detailed analysis of such high Mach number structures will be given elsewhere.

\section{CONCLUSIONS}

We believe that several very instructive results have emerged from the present study. The development of an ion-ion streaming instability in the presence of a strong magnetic field was followed through its early stages until nonlinear saturation. Predictions of the quasilinear moment equations and arguments for nonlinear stabilization by ion trapping were found to agree with the results of the simulation. One can therefore apply, with more confidence, similar simple theoretical considerations in describing more complex systems.

The scaling laws for this particular ion-ion instability were found for a variety of conditions, including the stabilization level of the field fluctuations. Finally, the ion interaction found for distributions of the form in Fig. 8 should be pertinent to the existence of double structure high Mach number shock waves, when the local Mach number falls in the range $M_{A}^{2}(x)<4[1+\beta(x)]$.
* Permanent address: University of Maryland, College Park, Maryland 20742.

$\dagger$ Alfred P. Sloan Research Fellow, 1970-72.

$\ddagger$ Permanent address: Plasma Physics Laboratory, Princeton University, Princeton, New Jersey 08540.

${ }_{1}$ D. A. Tidman and N. A. Krall, Shock Waves in Collisionless

Plasmas (Interscience, New York, 1971), Chap. 8.

${ }^{2}$ T. E. Stringer, J. Nucl. Energy Pt. C 6, 267 (1964).

${ }^{3}$ R. C. Davidson, N. A. Krall, K. Papadopoulos, and R. Shanny, Phys. Rev. Letters 24, 579 (1970).

40 . Buneman, Phys. Rev. 115, 503 (1959)

${ }^{5} \mathrm{~W}$. Manheimer, Phys. Fluids (to be published).

${ }^{6}$ J. M. Dawson, C. G. Hsi, and R. Shanny, in Plasma Physics and Controlled Nuclear Fusion Research (International Atomic Energy Agency, Vienna, 1969), Vol. 1, p. 735.

${ }^{7}$ R. L. Morse and C. Nielsen, Phys. Fluids 12, 2418 (1969). C. K. Birdsall, NASA Report No. SP-153 (1967) and references therein.

8 J. P. Boris, J. M. Dawson, J. H. Orens, and K. V. Roberts, Phys. Rev. Letters 25, 706 (1970).

${ }^{9}$ D. G. Bald win and G. Rowlands, Phys. Fluids 9, 2444 (1966).

${ }^{10} \mathrm{~T}$. Stix, The Theory of Plasma Waves (McGraw-Hill, New York, 1962), Chap. 1.

${ }_{11}$ D. W. Ross, Phys. Fluids 13, 746 (1970).

12 W. Crevier, Ph.D, thesis, University of Maryland (1969); W. Crevier and D. A. Tidman, Phys. Fluids 13, 2275 (1970).

${ }^{13}$ A. G. Es'kov, R. Kh. Kurtmulaev, A. I. Malyutiu, V. I. Pil'skï', and V. N. Servenov, Zh. Eksp. Teor. Fiz. 56, 1480 (1969) [Sov. Phys. JETP 29, 793 (1969)].

\title{
Resonance Cones in the Field Pattern of a Radio Frequency Probe in a Warm Anisotropic Plasma
}

\author{
R. K. Fisher* aNd R. W. GouLd ${ }^{\dagger}$ \\ California Institute of Technology, Pasadena, California 91109
}

(Received 29 June 1970)

\begin{abstract}
An experimental and theoretical investigation of the angular distribution of the electric field of a short radio frequency probe in a plasma in a magnetic field is described. The field is observed to become very large along a resonance cone whose axis is parallel to the static magnetic field and whose opening angle is observed to vary with incident probe frequency, cyclotron frequency, and plasma frequency in agreement with simple cold plasma dielectric theory. The relationship of these cones to the limiting phase- and group-velocity cones which appear in the theory of plane wave propagation is discussed. The addition of electron thermal velocities (warm plasma effects) is examined in the limit of a large static magnetic field. A fine structure appears inside the cones and is shown to result from an interference between a fast electromagnetic wave and a slow plasma wave. This interference structure is observed experimentally and measurements of the angular interference spacing are shown to agree with the warm plasma theory. The use of measurements of the resonance cones and structure as a diagnostic tool to determine the plasma density and electron temperature in a plasma in a magnetic field is discussed.
\end{abstract}

\section{INTRODUCTION}

The electromagnetic fields and radiation from sources in a plasma in a magnetic field have been the subject of many theoretical studies ${ }^{1-4}$ and have become of practical interest in connection with investigations employing rocket and satellite vehicles.

Much of the theoretical interest has centered on the small dipole antenna because of its inherent simplicity. The analysis of an oscillating point dipole in a cold anisotropic plasma ${ }^{3}$ shows that the fields should be- come singular along a cone whose axis is parallel to the static magnetic field and whose opening angle $\theta_{c}$ is determined by the plasma density, magnetic field strength, and incident frequency (see Fig. 1). It has been shown that the Poynting vector is also singular on the resonance cones, ${ }^{3}$ so that the total power flow and hence the radiation resistance is infinite for the point dipole antenna. This result, sometimes referred to as the "infinity catastrophe," has stirred considerable controversy. ${ }^{5}$ It has been proposed that the inclusion of effects such as electron collisions, ${ }^{b}$ electron 
thermal velocities, ${ }^{7,8}$ and sources of nonzero dimensions ${ }^{9}$ cause the fields along the cones (and hence the radiation resistance) to remain finite.

Considering the extent of the theoretical work on radiation from sources in anisotropic plasmas and the interest shown in the resonance cones, there has been remarkably little experimental activity in these areas. This paper describes an investigation of the angular field pattern of a short radio-frequency probe in a plasma in a magnetic field. These measurements, first reported in a previous paper, ${ }^{10}$ represent the first experimental verification of the existence of resonance cones along which the observed fields become very large. Further investigations have resulted in observations of a warm plasma fine structure ${ }^{8}$ in the angular field pattern near the resonance cones, resulting from an interference between a fast electromagnetic wave and a slow plasma wave. In this paper we describe both the experimental measurements and a quasistatic theory leading to the resonance cones and structure in more detail, extend the previous measurements, and discuss more fully the warm plasma theory leading to the observed interference structure. A more extensive treatment of the warm plasma theory, using the complete Maxwell equations and including a calculation of radiation resistance has been completed by Singh and Gould. ${ }^{11}$

The theory for a cold plasma is discussed in Sec. II. Section III describes the experimental apparatus and measurements of the resonance cone angle as a function of incident frequency and magnetic field strength. In Sec. IV, the relationship of these cones to the limiting phase- and group-velocity cones which appear in the theory of plane wave propagation is discussed. ${ }^{12} \mathrm{Sec}-$ tion $\mathrm{V}$ describes the warm plasma theory and measurements of the interference fine structure in the angular field pattern. Section VI presents some conclusions and discusses the use of the resonance cone angle as a diagnostic tool to measure the plasma density and the electron temperature in a plasma in a magnetic field.

\section{THEORY FOR A COLD ANISOTROPIC PLASMA}

Consider a probe with charge distribution $\rho_{\mathrm{ext}}$ oscillating at frequency $\omega$ in an infinite homogeneous collisionless plasma with an applied static magnetic field $\mathbf{B}_{0}=B_{0} \hat{z}$. The near-zone fields $(r \ll c / \omega)$ may be derived using the quasistatic approximation $E=-\nabla \varphi$. We must solve Poisson's equation $\boldsymbol{\nabla} \cdot \mathbf{D}=\rho_{\text {ext }}$ where $\mathbf{D}=\epsilon_{0} K \cdot \mathbf{E}$ and $\mathrm{K}$ is a tensor with $K_{x x}=K_{y y}=K_{\perp}, K_{x y}=-K_{y x}=K_{H}$, and $K_{z z}=K_{||}$as its nonzero components. For a cold plasma, neglecting terms of order $m_{e} / m_{i}$, we have

$$
K_{\perp}=1-\omega_{p}^{2} /\left(\omega^{2}-\omega_{c}^{2}\right) \text { and } K_{||}=1-\omega_{p}^{2} / \omega^{2} \text {, }
$$

where $\omega_{p}$ and $\omega_{c}$ are the electron plasma and cyclotron frequencies, respectively. These expressions are valid for frequencies $\omega$ large compared with $\Omega_{p}$ and $\Omega_{c}$, the ion plasma and cyclotron frequencies.
For simplicity, we will solve for the potential of an oscillating point charge located at the origin, $\rho_{\text {ext }}=$ $q \exp (-i \omega t) \delta(\mathbf{r})$. Although this is a nonphysical charge distribution, the solution we obtain with $q=1$ can be thought of as the Green's function for the problem and is therefore useful in obtaining the solution for more complex charge distributions. Also the main physical ideas are apparent from the Green's function. Taking the Fourier transform of Poisson's equation, we obtain an algebraic equation for the Fourier transform of the potential, and the inverse Fourier integral yields

$$
\varphi=\frac{q \exp (-i \omega t)}{\epsilon_{0}} \iiint \frac{\exp (i \mathbf{k} \cdot \mathbf{r})}{k_{\mid{ }^{2}} K_{\|}+k_{\perp} K_{\perp}} \frac{k_{\perp} d k_{\perp} d k_{\| \mid} d \phi}{(2 \pi)^{3}} .
$$

For a cold plasma, since $K_{||}$and $K_{\perp}$ are not functions of $\mathbf{k}$, the integrations can be performed directly, ${ }^{13}$ yielding

$$
\varphi(\rho, z)=\frac{q \exp (-i \omega t)}{4 \pi \epsilon_{0}\left(K_{\perp}{ }^{2} K_{11}\right)^{1 / 2}\left[\rho^{2} / K_{\perp}+z^{2} / K_{11}\right]^{1 / 2}}
$$

in cylindrical coordinates. ${ }^{14}$ Note that this is very similar to the potential of an oscillating charge in free space except that the square of the coordinates $\rho^{2}$ and $z^{2}$ are scaled by the appropriate dielectric constant in that coordinate direction.

To solve for the potential and fields of an oscillating dipole we merely take the derivative with respect to the appropriate spatial coordinate. All spatial derivatives of Eq. (3) will also have a singularity along the cone defined by the vanishing of the denominator,

$$
K_{\text {// }} \sin ^{2} \theta+K_{\perp} \cos ^{2} \theta=0,
$$

where $\theta=\tan ^{-1} \rho / z$ is the polar angle in spherical coordinates. As pointed out previously, the potential (3) with $q=1$ can be thought of as the Green's function for the problem and thus the resonance cones will exist regardless of the charge distribution on the ex-

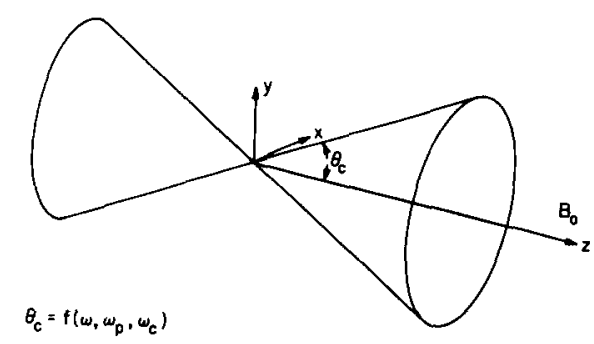

FIG. 1. Diagram showing resonance cones for a small source located at the origin. The axis of the cones is parallel to the static magnetic field $\boldsymbol{B}_{0}$ and the opening angle $\theta_{c}$ is determined by the plasma frequency $\omega_{p}$, cyclotron frequency $\omega_{c}$, and incident frequency $\omega$. 
citing probes. The singularity is a property of the medium rather than of the source. From (3), the cones exist only in frequency regions where either $K_{\| 1}$ or $K_{\perp}$ becomes negative, but not both. In a magnetoplasma these regions are $\omega$ less than both $\omega_{p}$ and $\omega_{c}$, hereafter called the "lower branch" $\left(0<\omega<\omega_{p}, \omega_{c}\right)$, and $\omega$ greater than both $\omega_{p}$ and $\omega_{c}$ but less than the upper hybrid frequency $\omega_{\mathrm{uh}}=\left(\omega_{p}^{2}+\omega_{c}^{2}\right)^{1 / 2}$, hereafter called the "upper branch" $\left(\omega_{p}, \omega_{c}<\omega<\omega_{\mathrm{uh}}\right)$. From Eq. (4) the resonance cone angle is given by

$$
\sin ^{2} \theta=\frac{\omega^{2}\left(\omega_{p}^{2}+\omega_{c}^{2}-\omega^{2}\right)}{\omega_{p}^{2} \omega_{c}^{2}} .
$$

Note that it is symmetric with respect to $\omega_{p}$ and $\omega_{c}$.

A calculation of the fields of an oscillating point dipole in a cold anisotropic plasma based on the full set of Maxwell's equations ${ }^{3}$ shows that in the far zone $(r \gg c / \omega)$ the fields also become singular at this same cone angle. The validity of the quasistatic solution presented here is not simply that the near-zone condition $(r \ll c / \omega)$ apply. We are dealing with a resonance (index of refraction $n \rightarrow \infty$ ) where electrostatic approximations are usually valid. The quasistatic solutions for the electric field are valid near the resonance cone (see Appendix A).

It should be noted that inclusion of the ion terms in the expressions for the plasma dielectric tensor would predict similar cones at frequencies $\omega<\Omega_{c}$, the ion cyclotron frequency, although no attempt was made to observe these "ion branch" cones experimentally.

\section{EXPERIMENTAL MEASUREMENTS AND DISCUSSION}

The experiment was performed in a steady-state argon rf discharge (pressure $\simeq 1 \mu$ ) in the apparatus illustrated in Fig. 2. The plasma was contained in a glass cylinder (diameter $\simeq 15 \mathrm{~cm}$ ) and the active discharge region was about $25 \mathrm{~cm}$ long. A static magnetic

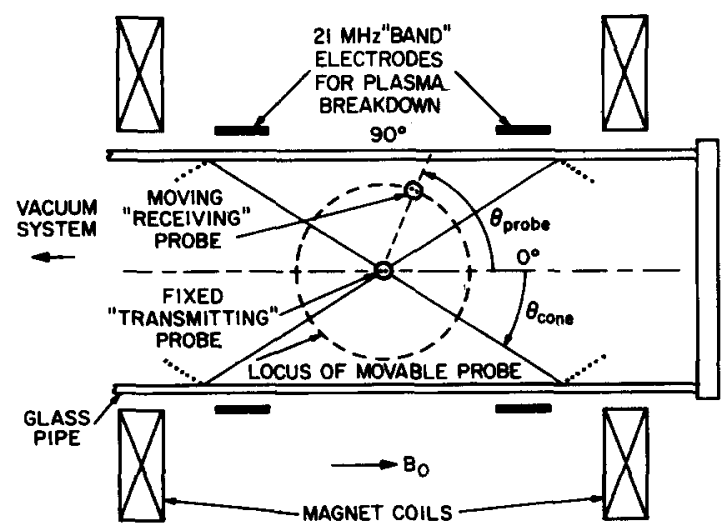

FrG. 2. Schematic of experiment to measure resonance cones and structure.

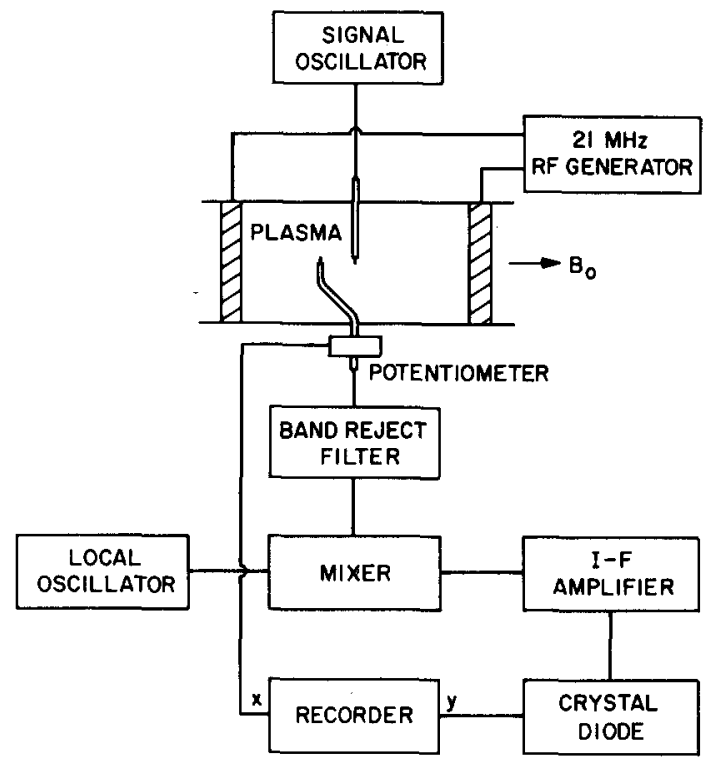

FIG. 3. Block diagram of experimental electronics.

field was produced along the axis of the plasma column by a pair of Helmholtz coils.

The transmitting and receiving probes used to study the resonance cones were introduced into the plasma through two ports on opposite sides of the glass cylinder. The probes were constructed from 0.070 in. or 0.087 in. o.d. semirigid $50 \Omega$ coaxial cable. The radiating portion of the probes consisted of approximately $2 \mathrm{~mm}$ of exposed center conductor. The transmitting probe was fixed in the center of the plasma column. A second probe was used to measure the fields set up by the transmitting probe. This receiving probe was constructed to rotate in a circular arc whose center is at the transmitting probe.

A block diagram of the experimental electronics is shown in Fig. 3. The plasma was produced by a 21 $\mathrm{MHz}, 100 \mathrm{~W}$ rf generator which was capacitively coupled to the plasma by means of a tuned $L C$ circuit, where part of the capacitance was furnished by two copper sleeves which fitted tightly around the glass cylinder. The signal into the transmitting probe was provided by a variable frequency uhf oscillator. The signal measured by the receiving probe was mixed with a local oscillator signal and fed into an $I-F$ amplifier whose output was rectified by a crystal diode and drove the $Y$ axis of an $X-Y$ recorder. A band reject filter was inserted before the mixer to reduce the $21 \mathrm{MHz}$ signal and its first harmonic present in the plasma. The $X$ axis of the recorder was controlled by a potentiometer which measured the angular position of the rotating motor-driven receiving probe with respect to the direction of the static magnetic field.

If the incident frequency of the transmitting probe 


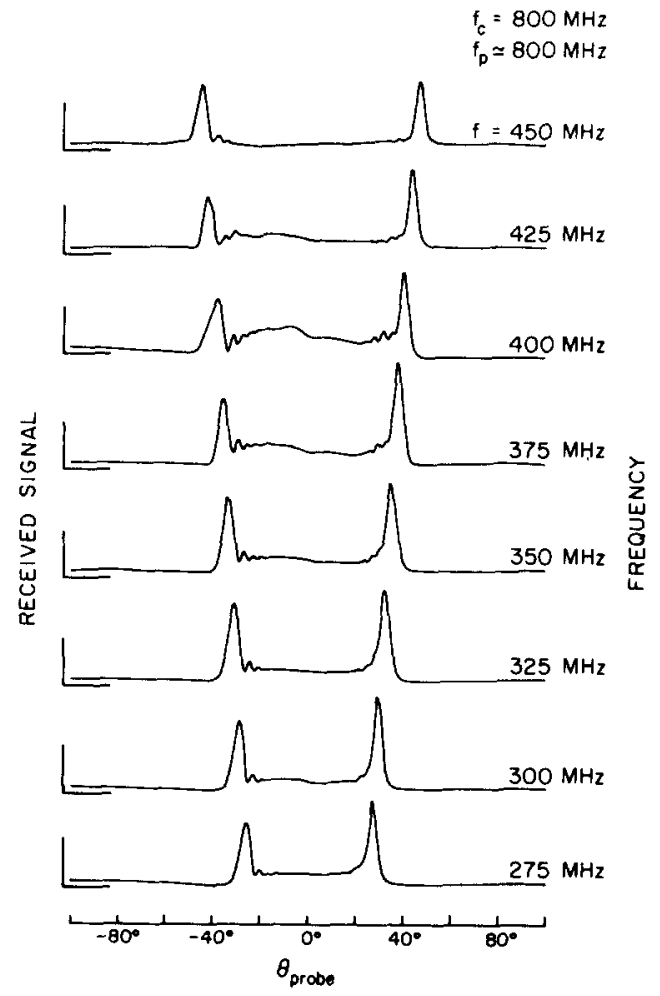

FIG. 4. Series of experimental traces showing variation of resonance cone angle with probe frequency for constant plasma density and constant magnetic field strength.

is chosen to be in a frequency range where cones exist, we would expect to see an increase in the received signal as the angle $\theta_{p}$ which the receiving probe position makes with respect to the magnetic field passes through the angle $\theta_{c}$ of the resonance cones set up by the transmitting probe.

\section{A. Cone Angle Location Measurements}

A series of traces taken at fixed plasma frequency $f_{p}$ and cyclotron frequency $f_{c}$ is shown in Fig. 4 . In each trace, the vertical axis is the received signal from the $I-F$ amplifier (roughly a linear power scale) and the horizontal axis is the probe location $\theta_{p}$, the angle the position of the receiving probe makes with respect to the magnetic field direction. The traces were taken at various incident frequencies and are displaced vertically for display purposes. The received signal is observed to become very large at the resonance cone angle. This is part of the lower branch since, as the frequency increases, the resonance cone angle increases in accord with Eq. (5). When the cone angle exceeded $60^{\circ}$, multiple reflections from the glass walls (the dotted lines in Fig. 2 illustrate reflected cones) complicated the traces. The fine structure which was observed to appear just inside the cones will be discussed in Sec. V.
If the experimentally measured cone angles are plotted versus the ratio $\omega / \omega_{c}$, we obtain a measure of the plasma frequency as shown in Fig. 5. The solid curves are plots of the lower and upper branch cone angle-frequency relationships predicted by $\mathrm{Eq}$. (5) for the indicated values of the parameter $\omega_{p} / \omega_{c}$. The circular points represent experimental data taken at a magnetic field corresponding to a cyclotron frequency $f_{c}=800 \mathrm{MHz}$, while the triangular points were taken at a lower magnetic field $\left(f_{c}=480 \mathrm{MHz}\right)$. The two sets of data indicate a plasma density corresponding to $\omega_{p} / \omega_{c} \simeq 1$ and 1.5 (or $n_{e} \simeq 8 \times 10^{9} \mathrm{~cm}^{-3}$ and $6.5 \times 10^{9} \mathrm{~cm}^{-3}$, respectively). An independent measurement using a $10.5 \mathrm{GHz}$ microwave interferometer ${ }^{15}$ (not shown in Figs. 2 or 3) confirms these electron densities to within $20 \%$. The data taken at the lower magnetic field show the upper and lower branches clearly separated by a frequency band in which, as predicted no cones were present. The above results show that the reasonance cone angle can be used as a diagnostic measure of the plasma density in a plasma in a magnetic field.

The agreement between experiment and theory shown in Fig. 5 appears to indicate that the plasma density radial profile was relatively uniform in the experimental plasma column, at least out to a radius equal to the probe separation in those measurements (approximately $6 \mathrm{~cm}$ ). As a check, the cone angles were measured with four different receiving probes, each at different radii (approximately $1,2,3$, and $6 \mathrm{~cm}$ ), and most of the measurements agree to within $2-3^{\circ}$, indicating that the plasma density was uniform to within $10-20 \%$ in this region.

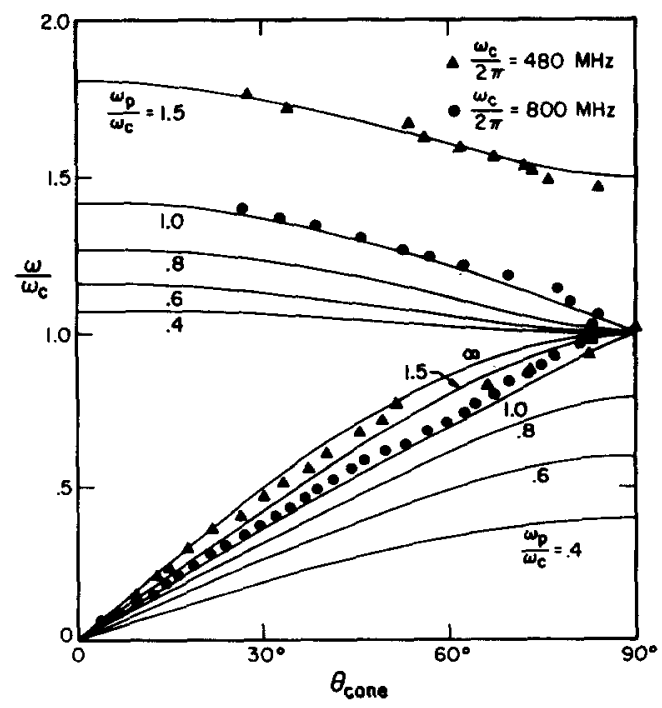

FIG. 5. Graph showing the experimentally observed location of the resonance cone angle versus the ratio $\omega / \omega_{c}$. Solid curves are those predicted by Eq. (5) for labeled values of $\omega_{p} / \omega_{c}$. 


\section{B. Phase and Amplitude Measurements}

Measurements of the relative phase of the received signal, as well as more quantitative measurements of the amplitude, were made using a second detection system. Here, the signal from the receiving probe was fed into the test channel of a Hewlett-Packard 8410A network analyzer with an $8413 \mathrm{~A}$ phase-gain indicator plug-in module. This instrument measured the amplitude (on a logarithmic scale) and phase of the received signal relative to a reference channel whose input was provided by a directional coupler connected to the signal source. A typical result is shown in Fig. 6. The resonance cone amplitude was usually $10-20 \mathrm{~dB}$ above the lowest level signal. The signal level inside the resonance cones was observed to be larger than that outside the cones for lower branch cone angles less than about $40-50^{\circ}$, but as the cone angle was increased further, this difference in levels slowly vanished.

The phase of the received signal changed markedly as the receiving probe passed through the resonance cone, the magnitude of this phase shift varying from $150^{\circ}$ to $400^{\circ}$ depending on $\omega, \omega_{c}, \omega_{p}$, and the separation between the probes. ${ }^{16}$ These phase measurements gave the first indication that the probes used in these experiments did not behave as simple dipoles. If the probes were simple dipoles, the receiving probe would measure the electric field of the transmitting probe. The nearzone field of an oscillating dipole can be obtained by taking two spatial derivatives of Eq. (3), one in the direction of the dipole to obtain the dipole potential and one in the direction of the desired component of
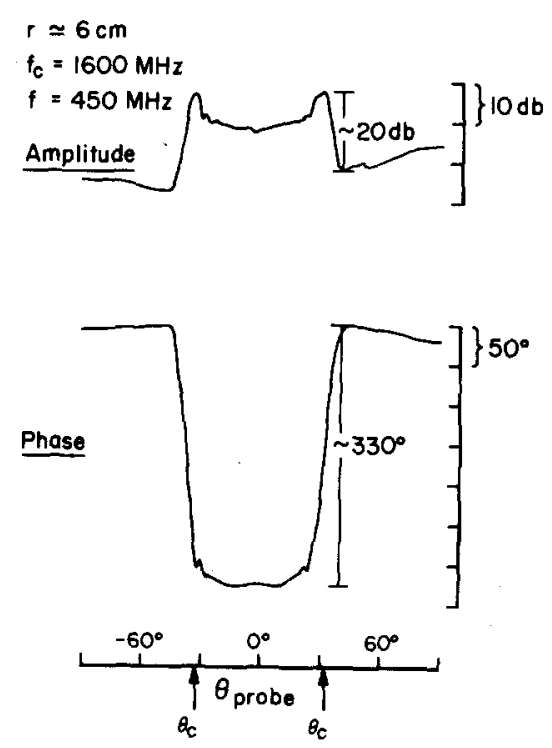

FIG. 6. Experimental traces showing relative amplitude on a logarithmic scale and phase of received signal versus $\theta_{\text {probe }}$ for $r=6 \mathrm{~cm}, f_{c}=1600 \mathrm{MHz}$, and $f=450 \mathrm{MHz}$. The signal undergoes a large phase shift as $\theta_{\text {probe }}$ passes through the resonance cone angle $\theta_{c}$. the electric field. This would result in an electric field proportional to $r^{2} / R^{5}$ where $r=\left(\rho^{2}+z^{2}\right)^{1 / 2}$ and $R=$

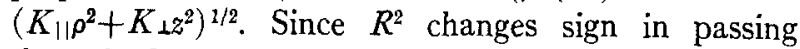
through the resonance cone, the phase of the electric field changes discontinuously by $5 / 2\left(180^{\circ}\right)=450^{\circ}$. The addition of electron collisions or electron thermal velocities to the theory would make the phase change gradual and must be considered to resolve ambiguities of $360^{\circ}$ in the phase shift. Considering a $z$-directed dipole in a cold uniaxial plasma for simplicity, the electric field at an arbitrary distance can be shown $n^{17}$ to contain terms proportional to $r^{2} / R^{3}, r^{2} / R^{4}$, and $r^{2} / R^{5}$. This would mean a phase shift anywhere between $270^{\circ}$ and $450^{\circ}$ depending on the size of $\omega R / c$ whose magnitude varied from zero on the resonance cone to infinity near $\omega=0$ and $\omega=\omega_{c}$. The experimental results were not consistent with the above dipole theory. Several attempts were made to construct true dipole antennas but these were unsuccessful. ${ }^{18}$ In Sec. V the addition of electron thermal velocities to the theory shows that there are really two waves contributing to the received signal, a fast electromagnetic wave and a slow plasma wave. The phase of each of these waves changes independently with angle and the interference between the waves causes the total signal to become very small at times. The total phase shift in passing through the resonance cone will depend on the relative amplitude of the slow and fast waves which depends on the damping in the plasma and sheath effects around the probes, making comparisons of theory and experiment very difficult. A calculation of the fields of an oscillating dipole in a warm uniaxial plasma ${ }^{11}$ has resulted in angular phase patterns which are similar to the experimental traces.

In Sec. II it was shown that the cones should exist regardless of the charge distribution on the exciting probe. In the attempts to construct true dipole probes, a variety of probe designs and orientations were investigated and the qualitative features of the resonance cones were always observed, as expected.

\section{PHASE AND GROUP VELOCITY IN AN ANISOTROPIC PLASMA}

The theory of plane wave propagation in an anisotropic plasma has been widely discussed ${ }^{19-21}$ The index of refraction $\mathbf{n}=\mathbf{k} c / \omega$ and wave vector $\mathbf{k}$ become singular at a certain angle $\psi_{c}$ which the wave vector makes with respect to the static magnetic field direction, and this angle is sometimes also referred to as the resonance cone angle. This resonance angle $\psi_{c}$ is the complementary angle of the physical angle $\theta_{c}$, at which the fields of a small source in a magnetoplasma become singular. This difference is not widely appreciated $^{22}$ and is explained below in terms of the directions of the phase and group velocities of waves in an anisotropic plasma. 


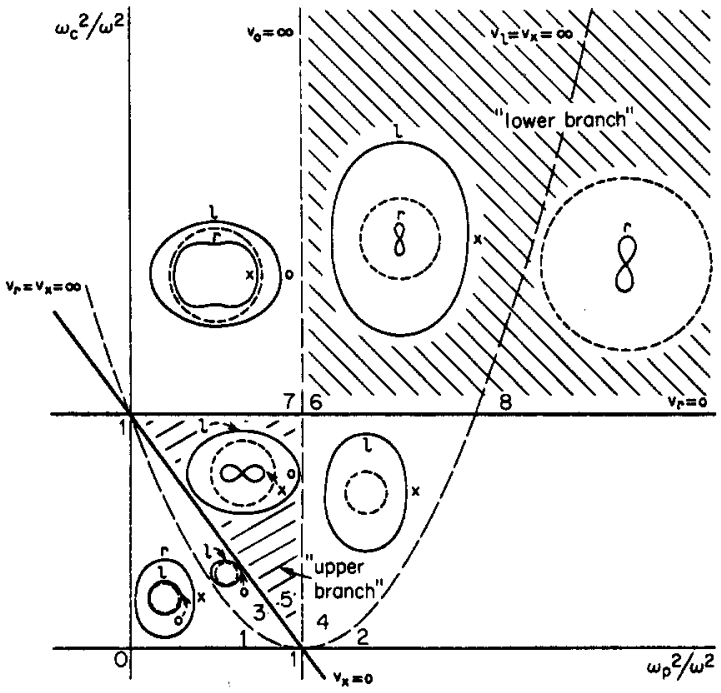

Fig. 7. Clemmow-Mullaly-Allis diagram for an electron plasma $\left(m_{\text {ion }} \rightarrow \infty\right)$. This two-dimensional parameter space is divided into different regions, and characteristic wave-normal surfaces are sketched for each region. The regions of interest are shaded.

In the study of plane wave propagation in an anisotropic plasma, the results are frequently presented in the form of a Clemmow-Mullaly-Allis diagram ${ }^{20}$ (see Fig. 7). For each region in this two-dimensional parameter space, cross sections of typical wave-normal surfaces are sketched. These sketches show the magnitude of the phase velocity $v_{p}$ versus the angle $\psi$ which the phase velocity makes with respect to the static magnetic field direction (which is vertical in these diagrams). There is azimuthal-symmetry about the magnetic field, and we see only a cross-section of a phase velocity surface. In the region corresponding to the lower branch of the cones $\left(\omega<\omega_{p}, \omega_{c}\right)$, regions 6 and 8 in Fig. 7, propagation is allowed only for $\psi<\psi_{c}$. Outside the limiting cone $\psi=\psi_{c}$ the waves are evanescent, i.e., there are no propagating waves. $\psi=\psi_{c}$ is called a resonance cone because the index of refraction $n \rightarrow \infty$ $\left(v_{p} \rightarrow 0\right)$ on the cone. In the region corresponding to the upper branch of the cones $\left(\omega_{p}, \omega_{c}<\omega<\omega^{\prime} u h\right)$, region 5 in Fig. 7 , propagation is allowed only for $\psi>\psi_{c}$. This interchange is due to the change in sign of both $K_{\perp}$ and $K_{\|}$for the plasma. On the other hand, Kuehl ${ }^{3}$ in discussing the fields of an oscillating point dipole in a uniaxial plasma $\left(B_{0} \rightarrow \infty\right.$, for which there is no upper branch), shows that for the lower branch $\left(\omega<\omega_{p}\right)$ the Poynting vector is nonzero only for $\theta<\theta_{c}$. So there is an apparent paradox in deciding the possible directions of propagation with respect to the magnetic field in a plasma. For example, consider a probe exciting waves of frequency $\omega=3^{1 / 2} \omega_{p} / 2$ in a plasma with $\omega_{0} \gg \omega_{p}$. The wave vector $\mathbf{k}$ of the waves, sometimes called the propagation vector, is real only at angles $\psi<\psi_{c}=30^{\circ}$ which the wave vector makes with respect to the magnetic field direction and the waves are evanescent for $\psi>30^{\circ}$. However, the Poynting vector is nonzero out to angles $\theta<\theta_{c}=60^{\circ}$ and, in fact, the field is largest at $\theta=60^{\circ}$, so there definitely is propagation at angles greater than $30^{\circ}$.

The resolution of this paradox is found if we remember that in an anisotropic plasma the directions of the phase velocity $\mathbf{v}_{p}=(\omega / k)(\mathbf{k} / k)$ and group velocity $\mathbf{v}_{g}=\partial \omega / \partial \mathbf{k}$ are generally different. Considering plane harmonic waves $\{$ wave fields $\exp [i(\mathbf{k} \cdot \mathbf{r}-\omega t)]$, Maxwell's curl equations becomes $\mathbf{k} \times \mathbf{E}=\omega \mathbf{B}$ and $\mathbf{k} \times \mathbf{B}=-\left(\omega / c^{2}\right) \mathbf{K} \cdot \mathbf{E}$. For simplicity, we limit our discussion to sufficiently large static magnetic fields so that, effectively, electrons move along field lines, i.e., $K_{\perp}=1$ and $K_{||}=1-\omega_{p}^{2} / \omega^{2}$. For plane waves whose wave vector (and hence phase velocity) makes an angle $\psi$ with respect to the magnetic field, $\mathbf{n}=\mathbf{k} c / \omega=$ ( $n \sin \psi, 0, n \cos \psi$ ) and the solution to the (dispersion) equation for the index of refraction $n$ is readily found to be

$$
n^{2}(\psi)=\left(\frac{c}{v_{p}}\right)^{2}=\frac{\left(1-\omega_{p}^{2} / \omega^{2}\right)}{\left[1-\left(\omega_{p}^{2} / \omega^{2}\right) \cos ^{2} \psi\right]} .
$$

When $\omega>\omega_{p}, n$ is real for all angles $\psi$, but when $\omega<\omega_{p}$, $n \rightarrow \infty$ (resonance) for $\psi=\psi_{c}=\cos ^{-1}\left(\omega / \omega_{p}\right)$. Furthermore for $\psi>\psi, n$ is imaginary (evanescent waves).

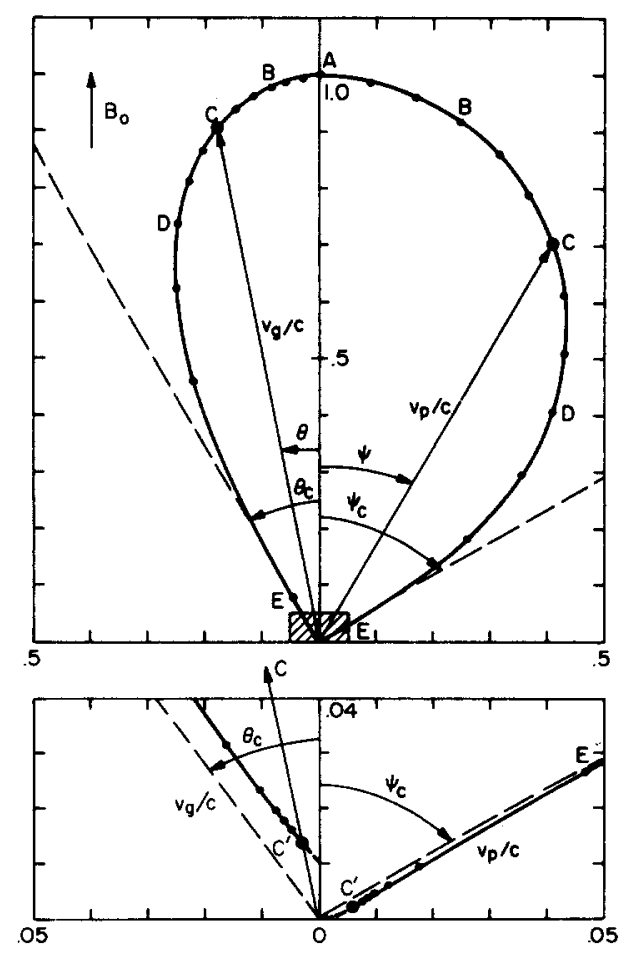

Fig. 8. Polar plot of phase velocity (right side) and group velocity (left side) for $B_{0}=\infty, \omega_{p} / \omega=2$. Lower plot shows shaded region, where electron thermal velocities are important, on an expanded scale. 
This behavior is illustrated in the right half of Fig. 8, which is a polar plot of $v_{p} / c=1 / n$ for $\omega=\omega_{p} / 2$ or $\psi_{c}=60^{\circ}$ (the dots correspond to $5^{\circ}$ increments in $\psi$ ). The angle $\psi_{c}$ defines a cone, whose axis coincides with the magnetic field, within which the phase velocity vector must lie and on which it tends to zero. We refer to this as the phase velocity cone. ${ }^{10}$

Using Eq. (6) we can obtain expressions for the magnitude of the group velocity associated with each $(\omega, \mathbf{k})$ and the angle $\theta$ which it makes with the magnetic field.

$$
\begin{gathered}
\left(\frac{v_{g}}{c}\right)^{2}=\frac{\left(1-\omega_{p}^{2} / \omega^{2}\right)\left[1-\left(\omega_{p}^{2} / \omega^{2}\right) \cos ^{2} \psi\right]}{1-2\left(\omega_{p}^{2} / \omega^{2}\right) \cos ^{2} \psi+\left(\omega_{p}^{4} / \omega^{4}\right) \cos ^{2} \psi}, \\
\tan \theta=-\tan \psi /\left(1-\omega_{p}^{2} / \omega^{2}\right) .
\end{gathered}
$$

Thus, the group velocity is not in the same direction as the phase velocity. In fact, the component of $\boldsymbol{\nabla}_{g}$ perpendicular to $B_{0}$ is opposite to the perpendicular component of $\mathbf{v}_{p}$. Near the resonance cone $(n \rightarrow \infty) \mathbf{v}_{g}$ and $\nabla_{p}$ are perpendicular (and coplanar with $\mathbf{B}_{0}$ ). This behavior is illustrated in the left half of Fig. 8, which is a polar plot of $v_{0} / c$. Each dot on the group velocity plot is to be associated with the corresponding dot on the phase velocity plot (e.g., A, B, C, ...). Equation (8) shows that $\theta$ increases monotonically with $\psi$ and reaches its maximum value $\theta_{c}$ at resonance $\left(\psi=\psi_{c}\right)$. Furthermore, $\psi_{c}+\theta_{c}=90^{\circ}$. Thus, $\theta_{c}$ defined a second cone, within which the group velocity vector must lie and on which it tends to zero. We refer to this as the group velocity cone. ${ }^{10}$ It can also be shown that the phase and group velocities are perpendicular on the resonance cone for a finite magnetic field. ${ }^{23}$

Although the group velocity vanishes as $\theta \rightarrow \theta_{c}$, the energy density and Poynting vector become singular, leading to the experimentally observed cones. Since propagation between two remote points in a plasma is determined by the group velocity, the importance of the group velocity and hence the group velocity cone rather than the customary phase velocity plots and cone should be emphasized. While this experiment was not performed in the far field region, the observed cone angles are those predicted by considerations of the group velocity and fields in the far zone.

\section{WARM PLASMA EFFECTS}

\section{A. Addition of Electron Thermal Velocities to the Theory}

We will again consider the quasistatic potential of an oscillating charge for simplicity. The solution obtained will be valid near the resonance cones. For a warm plasma $\left(2 k T_{c}=m v_{t h}{ }^{2}\right)$, the plasma dielectric constants $K_{\perp}$ and $K_{||}$become functions of the wave vector $\mathbf{k}$ and the inverse Fourier integral for the potential [Eq. (2)] could most easily be evaluated in the limit

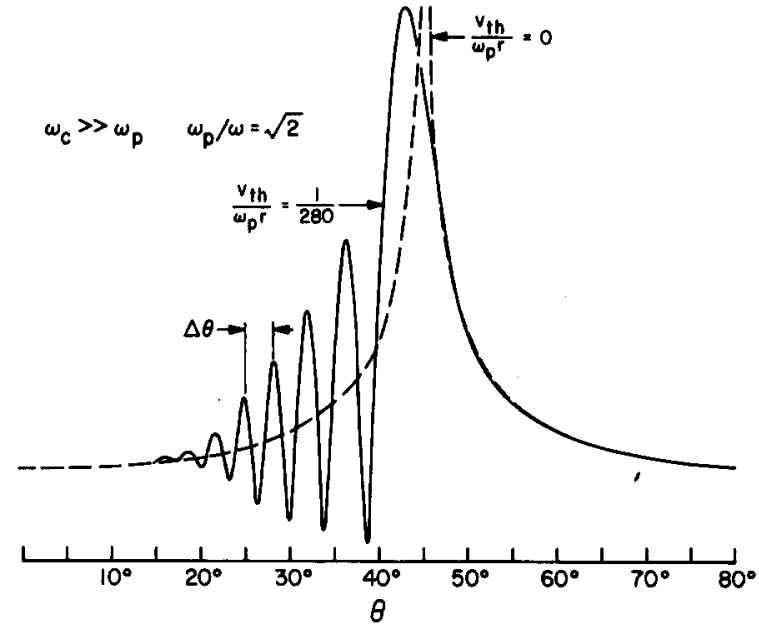

Fig. 9. Calculated angular dependence of the potential $|\varphi(\theta)|$ for $v_{t h} / \omega_{p} r=1 / 280$ (solid curve) and for $v_{t h} / \omega_{p} r=0$ (dashed curve).

of a large static magnetic field $\left(B_{0} \rightarrow \infty\right)$. Experimentally, this condition corresponds to $\omega_{c} \gg \omega_{p}, \omega$. In this limit the electrons in the plasma are essentially confined to the magnetic field lines and neither $K_{\perp}$ nor $K_{\|}$ depends on $k_{\perp}$,

$$
K_{\perp}=1 \text { and } K_{\| \mid}=1-\left(\omega_{p}^{2} / k_{\| \mid}^{2} v_{t h}^{2}\right) Z^{\prime}\left(\omega / k_{\| \mid} v_{t h}\right) \text {, }
$$

where $Z^{\prime}$ is the derivative of the plasma dispersion function ${ }^{24}$ with respect to its argument. The integrations over $\phi$ and $k_{\perp}$ can then be performed analytically, yielding

$\varphi(\rho, z)=\frac{q \exp (-i \omega t)}{4 \pi^{2} \epsilon_{0}} \int_{-\infty}^{\infty} K_{0}\left(k_{|| \rho} \rho K_{||^{1 / 2}}\right) \exp \left(i k_{\|} z\right) d k_{\|}$,

where $K_{0}$ is the modified Bessel function. This leaves only the $k_{||}$integration to be done numerically. Evaluation of this integral using an IBM 360 computer yields the cone structure shown in Fig. 9. (Note: a subroutine for calculating $Z^{\prime}$ is required.) Three important effects are observed. First, the potential now remains finite at the resonance cone. Second, the cone angle $\theta_{c}$ is shifted to a slightly smaller angle than that predicted by cold plasma theory $\left(\theta_{c}=\sin ^{-1} \omega / \omega_{p}\right)$. This is the probable explanation for the consistent trend evident in Fig. 5 where the experimental points seem to fall at a slightly smaller angle than that which would give the best agreement with theory, with the upper and lower branches both falling nearer to a given $\omega_{p} / \omega_{c}$ theoretical line. Third, and most interesting, an interference structure appears inside the cones. An empirical fit to the numerical data or evaluation of Eq. (10) by the method of stationary phase (see Appendix B) 
shows that the angular spacing between any two adjacent maxima in the interference structure is $\Delta \theta \propto$ $\left(v_{i h} / \omega_{p} r\right)^{2 / 3} \tan ^{1 / 3} \theta_{c}$ where $r$ is the distance between the probes. The angular spacing is largest between the resonance cone and the first maximum of the inter ference structure and then becomes smaller for the spacing between the first and second maxima and remains relatively constant, the spacing between adjacent maxima farther from the cone decreasing only slightly. A calculation of the fields of an oscillating dipole in a warm uniaxial plasma based on the full set of Maxwell's equations ${ }^{11}$ yields a similar relationship for the interference spacing.

This structure is due to an interference between a fast electromagnetic wave $\left(v_{\theta} \sim c\right)$ and a slow plasma wave $\left(v_{g} \sim v_{t h}\right)$. The left lower portion of Fig. 8 shows that the addition of electron thermal velocities modifies the group velocity polar plot so that inside the resonance cone $\left(\theta<\theta_{c}\right)$ for each direction $\theta$ there are two solutions, a fast wave (point $C$ on the figure) and a slow wave (point $C^{\prime}$ ). The right portion shows that the two waves also have different phase velocities (points $\mathrm{C}$ and $\mathrm{C}^{\prime}$ ) and that the phase velocity is now nonzero at all angles $\psi$. The phase of the slow wave changes rapidly with $\theta$, yielding the interference pattern. This is because near the cones the phase velocity is nearly perpendicular to the group velocity, i.e., the phase velocity is in the $\theta$ direction. In the electrostatic solution the slow wave is interfering with a fast "wave" which arrives instantaneously $(c \rightarrow \infty)$.

\section{B. Experimental Results}

The interference structure inside the cones was indeed observed experimentally and is present in Fig. 4. It was not as pronounced as shown in the theoretical curve in Fig. 9, but the amplitude of the interference structure depends on the relative amplitude of the slow and fast waves, which in turn depends on the type of probe exciting the waves (Fig. 9 is for the potential of an oscillating charge), the damping mechanisms in the plasma, ${ }^{11}$ and the sheaths around the probes, ${ }^{25}$ making quantitative amplitude comparisons of theory and experiment very difficult. The angular interference spacing is, however, dependent only on the relative phase of the slow and fast waves ${ }^{11}$ and does not depend significantly on the type of probe used.

The interference structure was also observed in the phase of the received signal near the cone angle (see Fig. 6). Under most experimental conditions the phase interference amplitude was relatively small, indicating that the plasma wave contribution to the total signal was small compared with the electromagnetic wave contribution. The measured angular interference spacing in the phase pattern was identical to that in the amplitude pattern, as expected. The interference was most prominent in both the phase and amplitude measurements at intermediate cone angles $\left(\theta_{c}\right.$ between approximately $30^{\circ}$ and $60^{\circ}$ ).

Having shown that the angular interference spacing could most easily be used to test the theory, detailed measurements of this spacing were undertaken. The static magnetic field was kept as large as possible $\left(\omega_{c} \gtrsim 2.3 \omega\right)$ to best approximate the limit $B_{0}=\infty$. An empirical fit to the numerical calculations of the integral given in Eq. (10) for the potential of an oscillating charge shows that the spacing between the first few successive maxima ${ }^{26}$ of the interference structure is given by

$$
\Delta \theta=5.8^{\circ}\left(\frac{200}{\omega_{p} r / v_{t h}}\right)^{2 / 3} \tan ^{1 / 3} \theta_{c} .
$$

Knowing the probe separation $r$ and the resonance cone angle $\theta_{c}$ [and hence the plasma frequency $\omega_{p}$ from Eq. (5)], the experimentally measured angular spacing $\Delta \theta$ and Eq. (11) can be used to find the electron thermal velocity $v_{t h}$ and hence the electron temperature $T_{e}$. From a large number of measurements of the angular spacing taken at various probe separations and at various cone angles, use of Eq. (11) indicated an electron temperature $T_{e}$ between 2 and $4 \mathrm{eV}$ with most measurements between 2 and $3 \mathrm{eV}$.

An independent measurement of the electron temperature was made using both cylindrical and planar

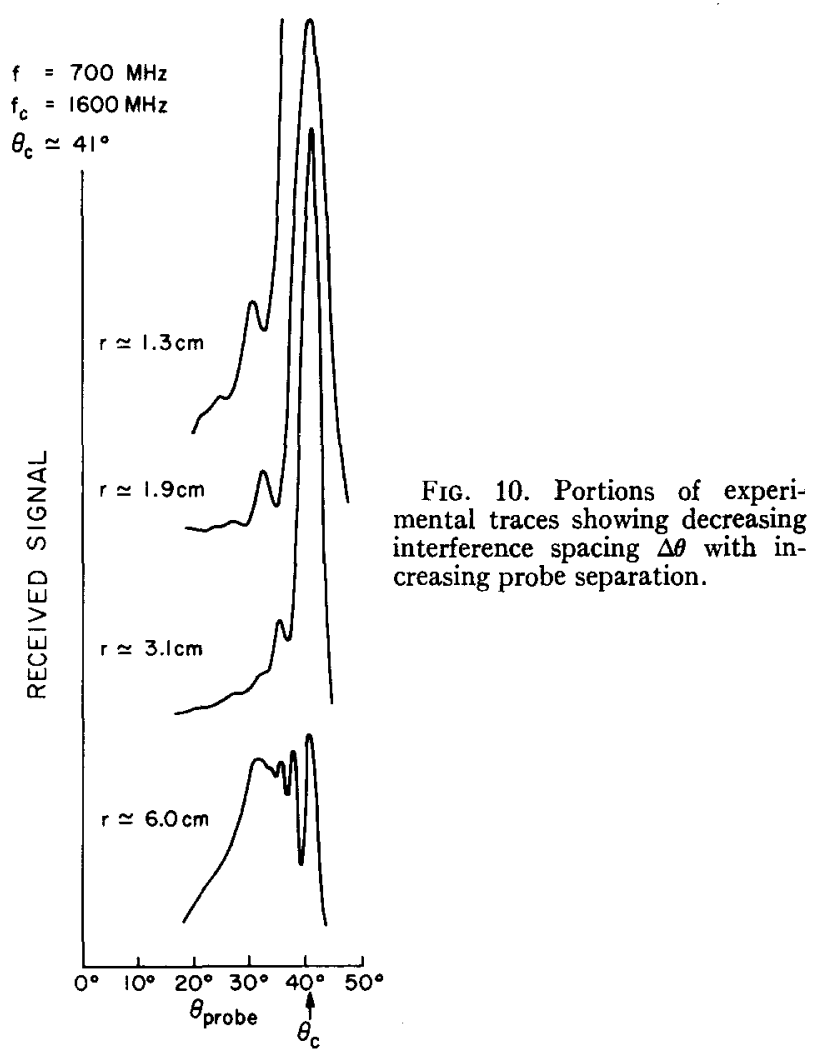


single Langmuir probes. Simple Langmuir probe theory fails when a strong magnetic field is present, but by using only the very small current portion of the currentvoltage probe characteristic, only those electrons within a few cyclotron radii are sampled and their collection is not substantially affected by the imposed static magnetic field. This has the disadvantage, however, of only sampling the high-energy tail of the electron distribution, and it is not certain that the energy distribution in the plasma was Maxwellian. These probe measurements yielded an electron temperature of about 3-5 eV. The planar probe yielded the most well-defined ion saturation current and probably the best measure of the electron temperature, about $3 \mathrm{eV}$, and this is in fairly good agreement with that calculated from the angular interference spacing.

To further test the theory, four receiving probes were constructed, each at a different radius from the transmitting probe in order to verify that $\Delta \theta \propto r^{-2 / 3}$. Typical experimental results showing the decreasing interference spacing with increasing probe separation are presented in Fig. 10. In Fig. 11 the triangular points are the measured angular spacing between the first maximum of the interference structure and the cone; the circular points that between the second maximum and the cone. Both sets of experimental points are consistent with the $r^{-2 / 3}$ dependence. Similar data, taken under a variety of plasma conditions, when plotted on a $\log -\log$ plot and fitted to a straight line by a least-squares procedure, yielded an average slope $-0.74 \pm 0.05$. This somewhat higher value than the expected $-\frac{2}{3}$ may be due to perturbations on the plasma due to the presence of the probes. If the slow plasma wave is assumed to be launched and detected at the edge of the probe sheaths, this would decrease the effective distance between the probes. Using a sheath thickness of five Debye lengths and replotting

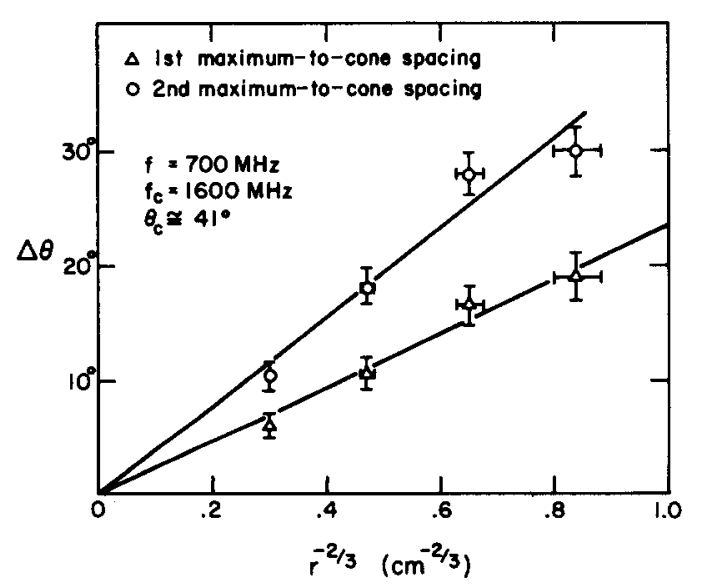

FIG. 11. Experimental interference spacing $\Delta \theta$ versus $r^{-2 / 3}$, where $r$ is the distance between the probes.

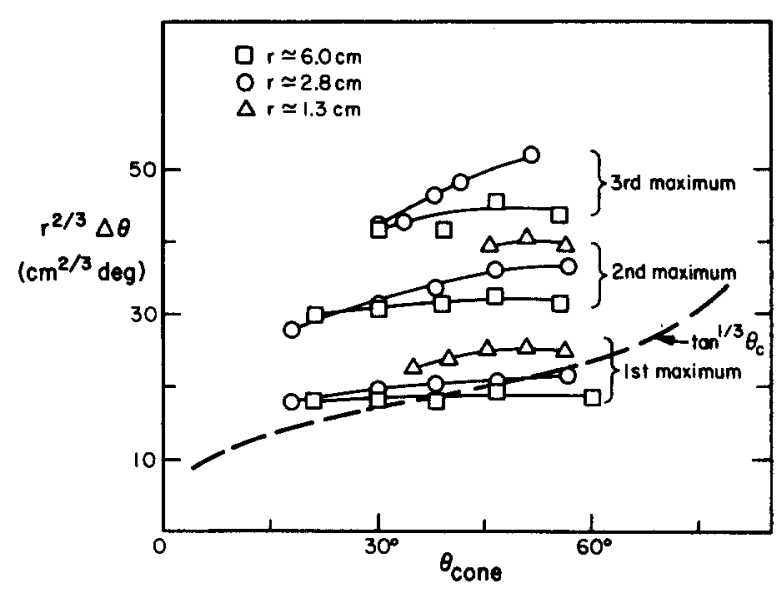

FIG. 12. Dependence of angular interference spacing on resonance cone angle. Vertical axis is $r^{2 / 3} \Delta \theta$ so that data taken at three different probe separations can be presented on a single plot. The dashed line is theoretical relationship $\Delta \theta \propto \tan ^{1 / 3} \theta_{c}$ on an arbitrary vertical scale. Data for the measured angular spacing between the first, second and third maxima and the cone are presented.

the data yields a corrected average slope $-0.70 \pm 0.04$. Another effect, and one which cannot easily be quantitatively taken into account, is that the average plasma density between two probes should decrease as the probe separation decreases. This would also cause the experimental slope to be too large.

Measurements of the interference spacing as a function of cone angle were also taken and the results are presented in Fig. 12. The vertical axis is $r^{2 / 3} \Delta \theta$ so that the data taken at three different probe separations could be presented on a single plot; the triangular points were taken with $r \simeq 1.3 \mathrm{~cm}$, the circular points with $r \simeq 2.8 \mathrm{~cm}$, and the square points with $r \simeq 6 \mathrm{~cm} .{ }^{27}$ The horizontal axis is $\theta_{c}$, the resonance cone angle. For each probe separation, data corresponding to the measured angular spacing between the first, second, and third maxima of the interference structure and the cone are shown (the location of the third maximum was not measurable for $r \simeq 1.3 \mathrm{~cm}$ ). For a given plasma density, electron temperature, and probe separation, the angular spacing should obey $\Delta \theta \propto \tan ^{1 / 3} \theta_{c}$ [see Eq. (11)]. The dashed line in Fig. 12 is a plot of $\tan ^{1 / 3} \theta_{c}$ on an arbitrary vertical scale to allow qualitative comparison of theory and experiment. The measured variation of $\Delta \theta$ with cone angle was, in general, somewhat flatter than $\tan ^{1 / 3} \theta_{c}$, especially for $r \simeq 6 \mathrm{~cm}$, perhaps indicating the need for a finite magnetic field theory.

\section{DISCUSSION}

This work represents the first experimental verification of the existence of the widely discussed resonance cones. The cones were shown to exist regardless of the charge distribution on the exciting probe and the 
cone angle was observed to vary with incident probe frequency, electron cyclotron frequency, and electron plasma frequency in agreement with simple cold plasma dielectric theory. It was noted that similar cones might be expected near the ion cyclotron frequency.

Measurement of the reasonance cone angle can be used as a diagnostic measure of the plasma density in a plasma in a magnetic field. Since the theory of Langmuir probes is not completely understood in the presence of a magnetic field, and since many plasmas are too small to be reliably analyzed using a microwave interferometer, the resonance cone measurement could be a very useful diagnostic tool. Very little equipment is required: an oscillator chosen in the proper frequency range, transmitting and receiving probes (which could both be entered through the same port), and a detection system (which could be as simple as a crystal detector and a dc voltmeter if the probe separation were small enough). The minimum distance between the probes is limited by the Debye length in the plasma and the loss in angular resolution as the probe separation decreases. Cone angle measurements have easily been made with probe separations as small as $1.3 \mathrm{~cm}$.

The addition of electron thermal velocities to the theory could be easily treated only in the limit of a large static magnetic field $\left(\omega_{c} \gg \omega_{p}, \omega\right)$. The fine structure observed near the resonance cones was shown to result from an interference between an electromagnetic wave and a plasma wave. The angular interference spacing is believed to be independent of the type and orientation of the probe exciting the waves, thus allowing quantitative comparisons of the theoretical and experimental fine structure spacing to be made. Measurements of this spacing yielded an electron temperature in agreement with a Langmuir probe measurement, indicating that the angular field measurements can be used as a diagnostic measure of the electron temperature as well as the plasma density as discussed previously. In addition, the interference spacing was observed to vary with probe separation in agreement with theory. There is a definite need for a warmplasma theory which is valid for a finite magnetic field, however, as indicated by the lack of complete agreement for the variation of the interference spacing with cone angle. A finite magnetic field theory would also allow investigation of the structure of the upper branch cones. Preliminary measurements indicate an interference structure which appears outside the resonance cones for the upper branch. This would be expected by analogy with the Clemmow-Mullaly-Allis diagram; propagation is allowed inside the cones for the lower branch, and outside the cones for the upper branch. A theory valid for a finite magnetic field would allow examination of the connection between the slow plasma waves described here and the cyclotron harmonic waves. ${ }^{28}$

\section{ACKNOWLEDGMENTS}

The authors gratefully acknowledge N. Singh for his work on theoretical aspects of this research.

This research was supported in part by the Office of Naval Research under Contract Nonr 220(50) and in part by the Atomic Energy Commission under Contract AT(04-3)-767. In addition, one of the authors (R.K.F.) received support as a National Science Foundation Graduate Fellow.

\section{APPENDIX A: VALIDITY OF THE QUASISTATIC APPROXIMATION}

The validity of the quasistatic solutions presented in Secs. II and V is not simply that the near-zone condition $(r \ll c / \omega)$ apply. Maxwell's equations allow the electric field of a source to be obtained from the vector and scalar potentials $\mathbf{E}=-\nabla \varphi-(1 / c) \partial \mathbf{A} / \partial t$. In using the electrostatic approximation, the contributions to the field from the vector potential are neglected. Considering a $z$-directed dipole in a uniaxial plasma $\left(B_{0} \rightarrow \infty\right)$ for simplicity, the electric field can be shown ${ }^{17}$ to contain terms proportional to $r^{2} / R^{5}, r^{2} / R^{4}$, and $r^{2} / R^{3}$ where $r=\left(\rho^{2}+z^{2}\right)^{1 / 2}$ and $R=\left(K_{\|} \rho^{2}+K_{\perp z^{2}}\right)^{1 / 2}$ which vanishes on the resonance cones. The only term which is important near the cone is the $r^{2} / R^{5}$ term which also arises from the quasistatic solution for the potential $\varphi$. Thus, the contributions from the vector potential are not important near the cone.

\section{APPENDIX B : DERIVATION OF FINE STRUCTURE SPACING BY METHOD OF STATIONARY PHASE}

Near the resonance cone we can use the large argument expansion of the modified Bessel function in Eq. (10), so that the potential of an oscillating charge in a warm uniaxial plasma becomes

$$
\begin{aligned}
\varphi(\rho, z)= & \frac{q \exp (-i \omega t)}{4 \pi^{2} \epsilon_{0}} \\
& \times \int_{-\infty}^{\infty}\left(\frac{\pi}{2 k_{\|} \rho K_{\|}{ }^{1 / 2}}\right)^{1 / 2} \exp \left[i \Phi\left(k_{\|}\right)\right] d k_{\|},
\end{aligned}
$$

where $\Phi\left(k_{||}\right)=k_{|| z} z i k_{||} \rho K_{||}^{1 / 2}$. Because of the rapidly oscillating character of $\exp \left[i \Phi\left(k_{11}\right)\right]$, the contributions to the integral from the neighborhood of $k_{\mid l}$ will largely cancel unless

$$
\Phi^{\prime}\left(k_{\|}\right)=z+i \rho K_{||^{1 / 2}}+i k_{\|} \rho K_{\|\left.\right|^{\prime}} / K_{||^{1 / 2}}=0 .
$$

The angular interference spacing can then be found using

$$
\varphi(\rho, z) \propto \exp \left[i \Phi\left(k_{\|_{0}}\right)\right],
$$

where $k_{110}$ is a root of Eq. (B2). In finding the roots of Eq. (B2), we use the low-temperature $\left(v_{t h} \ll \omega / k_{||}\right)$ 
expansion of the plasma dispersion function so that

$$
K_{\| \mid}=1-\frac{\omega_{p}^{2}}{\omega^{2}}\left(1+\frac{3 k_{1 \mid}^{2} \eta_{t h}^{2}}{2 \omega^{2}}\right) \text {. }
$$

Following the procedures outlined above with the assumptions that $v_{t h} \ll \omega / k_{||}$, and that $\theta_{c}-\theta$ is a small angle, we find that

$$
\Phi\left(k_{|| 0}\right) \propto\left(\omega_{p} r / v_{t h}\right)\left(\cot ^{1 / 2} \theta_{c}\right)\left(\theta_{c}-\theta\right)^{3 / 2}
$$

so that the angular interference spacing $\Delta \theta=\theta_{c}-\theta$ is given by

$$
\Delta \theta \propto\left(v_{t h} / \omega_{p} r\right)^{2 / 3} \tan ^{1 / 3} \theta_{c}
$$

which is the desired result.

\footnotetext{
* Present address: Mason Laboratory, Yale University, New Haven, Connecticut 06520.

† Present address: United States Atomic Energy Commission, Washington, D.C. 20545.

1 F. V. Bunkin, Zh. Eksp. Teor. Fiz. 32, 338 (1957) [Sov. Phys. JETP 5, 277 (1957) ]; H. Kogelnik, J. Res. Natl. Bur. Std. (U.S.) $64 \mathrm{D}, 515$ (1960).

2 B. P. Kononov, A. A. Rudhadze, and G. V. Solodukhov, Zh.

Tekh. Fiz. 31, 565 (1961) [Sov. Phys. Tech. Phys. 6, 405 (1961)].

${ }^{8}$ H. H. Kuehl, Phys. Fluids 5, 1095 (1962).

4 K. G. Balmain, IEEE Trans. Antennas Propagation AP-12, 605 (1964).

${ }^{6}$ H. Staras, Radio Sci. 1, 1013 (1966); K. S. H. Lee and C. H.

Papas, ibid. 1, 1020 (1966); D. Walsh and H. Weil, ibid. 1, 1025

(1966); K. S. H. Lee and C. H. Papas, ibid. 1, 1027 (1966).

T T. R. Kaiser, Planet. Space Sci. 9, 639 (1962).

${ }^{7}$ G. Deschamps and O. Kesler, Radio Sci. 2, 757 (1967); J.

Tunaley and R. Grard, Ann. Geophysics (France) 25, 55 (1969).

${ }^{8}$ R. K. Fisher and R. W. Gould, Phys. Letters 31 A, 235 (1970).

E. Arbel and L. B. Felsen, in Electromagnetic Theory and Antennas, edited by E. C. Jordan (Permagon, New York, 1963), p. 421 ; H. Staras, IEEE Trans. Antennas Propagation AP-12, 695 (1964).

${ }_{10}$ R. K. Fisher and R. W. Gould, Phys. Rev. Letters 22, 1093 (1969).
}

11 N. Singh and R. W. Gould, Bull. Am. Phys. Soc. 14, 1004 (1969) and Radio Sci. (to be published); N. Singh, Ph.D. thesis, California Institute of Technology (1971).

12 R. W. Gould and R. K. Fisher, in Proceedings of the Ninth International Conference on Phenomena in Ionized Gases (Institute of Physics, Academy of the Socialist Republic of Romania, Bucharest, Romania, 1969), p. 435.

${ }^{13}$ See, e.g., I. S. Gradshteyn and I. M. Ryzhik, Tables of Integrals, Series and Products (Academic, New York, 1965), integrals 8.411-1 (p. 952), 6.532-4 (p. 678), 6.678-1, 6.71-12 (p. 731), and 6.671-14 (p. 732)

${ }_{14}$ This result differs from that of Kononov et al. (Ref. 2). Their analysis of the near-zone fields is incorrect. The expression (in Eq. (I-3) ] for the fields outside the cones should be identical (i.e., nonzero) to that inside the cones.

${ }_{16} \mathrm{M}$. A. Heald and C. B. Wharton, Plasma Diagnostics with Microwaves (Wiley, New York, 1965), p. 117.

${ }^{16}$ For a more detailed discussion of the phase measurements, see R. K. Fisher, Ph.D. thesis, California Institute of Technology (1970).

${ }_{17}$ P. C. Clemmow, Proc. IEEE 110, 101 (1963).

18 R. K. Fisher, Ph.D. thesis, California Institute of Technology (1970).

${ }^{19} \mathrm{~W}$. P. Allis, S. J. Buchsbaum, and A. Bers, Waves in Anisotropic Plasmas (MIT Press, Cambridge, Mass. 1963), Chap. 3 .

${ }_{20} \mathrm{~T}$. H. Stix, The Theory of Plasma Wares (McGraw-Hill, New York, 1962), Chaps. 2 and 3.

${ }^{21}$ G. Bekefi, Radiation Processes in Plasmas (Wiley, New York, 1966), Chap. 1.

${ }^{22}$ The possibility of confusion is indicated in that Tunaley and Grard ${ }^{7}$, erroneously state that the fields of a source in an Inisotropic plasma should exhibit resonance cones at angle $\psi_{c}$. ${ }^{28}$ Reference 21, p. 13-14.

24 B. D. Fried and S. D. Conte, The Plasma Dispersion Function (Academic, New York, 1961), pp. 1-8.

${ }^{25}$ S. R. Seshadri, Proc. IEEE 113, 593 (1966).

${ }^{26}$ Note that this does not apply to the spacing between the first maximum and the resonance cone itself. The constant of proportionality is then $8.7^{\circ}$ rather than $5.8^{\circ}$.

${ }^{27}$ The data taken at different probe separations were taken on different days and $\omega_{p}$ and $v_{t h}$ were probably different for each probe separation and were not measured. This is the reason why the curves for $r \simeq 1.3,2.8$, and $6 \mathrm{~cm}$ do not fall closer together for a given maximum.

${ }_{28}$ R. S. Harp, in Proceedings of the Seventh International Conference on Phemomena in Ionized Gases (Gradevinska Knjiga, Belgrade, Yugoslavia, 1966), Vol. II, p. 294. 